\title{
Combined Modeling of Stellar Evolution and Pulsation
}

\author{
Z. Csubry ${ }^{1}$, R. Szabó ${ }^{1}$, Z. Kolláth ${ }^{1}$, J.R. Buchler ${ }^{2}$ \\ ${ }^{1}$ Konkoly Observatory, Budapest, HUNGARY \\ ${ }^{2}$ Physics Department, University of Florida, Gainesville, Florida, USA
}

\begin{abstract}
The radial pulsations of variable stars at specified points along their evolution are accurately described with numerical hydrodynamics. The behavior of the pulsations along evolutionary tracks can then be modeled with amplitude equations, whose coefficients are obtained from fits to these hydrodynamical models. To simulate stellar evolution we make the stellar core's properties time-dependent and can investigate directly the effects on pulsation despite the very different timescales (dynamical, thermal, evolutionary).
\end{abstract}

\section{Time-dependent amplitude equations}

Starting the hydrocode with different initial conditions at fixed stellar parameters $\left(M, L, T_{\text {eff }}\right)$, time-independent amplitude equations (AE) (Buchler \& Goupil, 1984) are fitted via the analytic signal method (Kolláth \& Buchler, 2001). The AEs' fixed point solutions determine the nature of the dynamical behavior of the model (Kolláth et al., 2001). By interpolating the AE coefficients for models with various $T_{\text {eff }}$ we can thus follow the instantaneous values of the amplitudes along an evolutionary path (say with a fixed luminosity), and thus simulate the evolution of the pulsational behavior through the entire instability strip.

The time-dependence of the AE coefficients is obtained from the $T_{\text {eff }}$-dependent coefficients by the rate of evolution along the evolutionary track. Integrating the time-dependent AEs, we obtain the evolution of the pulsation amplitude(s) of the model as it switches from one behavior to another, whether by soft or by hard bifurcation. It is found that there can be a substantial delay in the switching which is not necessarily on a thermal time-scale, but which can be either on an evolutionary or on a very extended thermal time-scale (Buchler \& Kolláth, 2001, BK).

\section{Evolutionary model}

Evolution effects were simulated in our hydrocode by changing the radius of the stellar core continuously, and with a varying energy production in the inner zones. This results in variations of the luminosity and $T_{\text {eff }}$ of the model. With precise calibration of these effects we can simulate the evolution of the envelope along an evolutionary track on the HR diagram.

We have applied this approach to an evolutionary RR Lyrae model with $0.65 M_{\odot}$ at the red edge of the first overtone $(\mathrm{O} 1)$ instability strip. Our goal was to investigate the switch-over to the fundamental $(\mathrm{F})$ mode. The calculation was accelerated by using an evolutionary rate $\left(d \ln T_{\text {eff }} / d t\right) 10$ times its real value. 

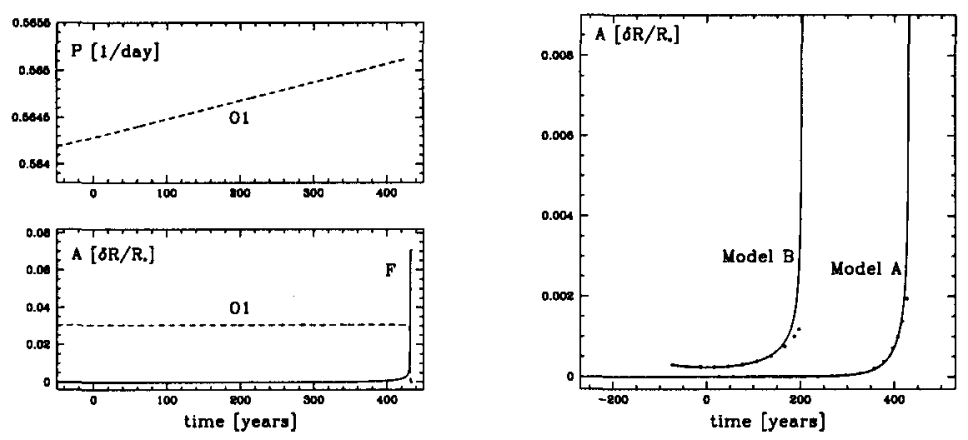

Figure 1. Left: Period and F amplitude evolution of model A along the whole calculation. Right: Evolution of $\mathrm{F}$ amplitudes (solid lines) and the fitted exponential variation (dotted lines) of model A and B.

For the sake of studying the transients, long initial calculations $(40,000$ years) with the pulsation suppressed by large viscosity and large time-step were needed. After that the static model was perturbed and a further 400 years (about 300,000 pulsations) were required, for the fundamental amplitude to vanish and for the first overtone to reach the limit cycle (model A). The period and amplitude evolution can be seen on the left of Fig. 1.

The approximate time when the first overtone limit cycle becomes unstable $\left(\tau_{0}\right)$ is calculated from AEs fitted to models computed at different epochs. The variation of the $\mathrm{F}$ amplitude $A$ is given by the solution of $d A / d t=\chi\left(t-\tau_{0}\right) A$ (i.e. $A=A_{0} \exp \left(\frac{1}{2} \chi t^{2}\right)$ ) when $A$ is small (Fig. 1, right). Even this very simple model gives a good prediction of the transition time. For the rate of change of the stability root we obtain $\chi \approx 8 \times 10^{-20}$. The transition time (400-500 years) agrees with the prediction of $\mathrm{BK}$ with the same $\chi$. For model $\mathrm{B}(\sim 0.0002$ initial amplitude) the transition time reduces to $\sim 200$ years.

We conclude that amplitude equations predict very well the temporal behavior of mode switching that is observed in very lengthy, full hydrodynamical calculations. The transition time here is $\sqrt{\chi^{-1}}$, where $\chi$ is the evolutionary rate of change of the stability root of the overtone mode $\kappa$. This time-scale is very large compared to the thermal time-scale, which is $\kappa^{-1}$.

This work was supported by Hungarian OTKA (T-026031) and NSF.

\section{References}

Buchler, J.R., \& Goupil, M.J. 1984, ApJ, 279, 394

Buchler, J.R., \& Kolláth, Z. 2001, Mode Switching Time-Scales in the Classical Variable Stars, A\&A, submitted

Kolláth, Z. \& Buchler, J. R. 2001, in Astrophysics and Space Science Library, Vol. 257, Stellar Pulsation - Nonlinear Studies, eds. M. Takeuti \& D. Sasselov, (Dordrecht: Kluwer Academic Publishers), 29

Kolláth, Z., Buchler, J.R., Szabó, R., Csubry, Z. 2001, Nonlinear Beat Cepheid and RR Lyrae Models, A\&A, submitted 